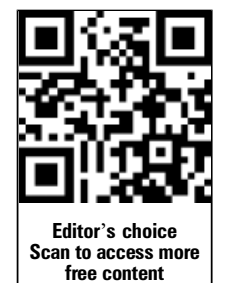

free content

- Additional data are published online only. To view this file please visit the journal online (http://dx.doi.org/10. 1136/bjsports-2013-092248)

For numbered affiliations see end of article.

\section{Correspondence to} Dr Paul McCrory, The Florey Institute of Neuroscience and Mental Health, Heidelberg, VIC 3084 Australia; paulmccrory@icloud.com

Received 25 January 2013 Accepted 26 January 2013

To cite: McCrory $P$, Meeuwisse WH, Kutcher JS, et al. Br J Sports Med 2013;47:327-330.

\title{
What is the evidence for chronic concussion-related changes in retired athletes: behavioural, pathological and clinical outcomes?
}

\author{
Paul McCrory, ${ }^{1}$ Willem H Meeuwisse, ${ }^{2,3}$ Jeffrey S Kutcher, ${ }^{4}$ Barry D Jordan, ${ }^{5}$ \\ Andrew Gardner ${ }^{6}$
}

\begin{abstract}
Objective The purpose of this paper was to review the current state of evidence for chronic traumatic encephalopathy (CTE) in retired athletes and to consider the potential differential diagnoses that require consideration when retired athletes present with cognitive and psychiatric problems.
\end{abstract}

Data sources MEDLINE, CINAHL, EMBASE, Mosby's Index, PsycEXTRA, PsycINFO and Scopus. Key words included CTE, dementia pugilistica, punch drunk syndrome, traumatic encephalopathy, CTE, repetitive head injury, sports concussion, multiple concussions, chronic concussions, subconcussive blow and sportsrelated traumatic brain injury.

Results At present, there are no published epidemiological, cross-sectional or prospective studies relating to modern CTE. Owing to the nature of the published studies, being case reports or pathological case series, it is not possible to determine the causality or risk factors with any certainty. As such, the speculation that repeated concussion or subconcussive impacts cause CTE remains unproven. The extent to which age-related changes, psychiatric or mental health illness, alcohol/drug use or coexisting dementing illnesses contribute to this process is largely unaccounted for in the published literature.

Conclusions At present, the interpretation of causation in the modern CTE case studies should proceed cautiously. The causal assumptions require further prospective or longitudinal studies on the topic.

\section{INTRODUCTION}

Considerable attention surrounds the potential for long-term problems in athletes with high exposure to head impacts during a career in collision sport. ${ }^{1}$ There is evidence ${ }^{2-4}$ supporting an association between long-term cognitive, neurobehavioural, psychiatric problems and participation in sport. ${ }^{5}$ Given that sports-related concussion is a common injury and that concussive or subconcussive blows to the head or body are an inevitable consequence of sports participation, if a causal relationship between these impacts and later-life neuropsychiatric disease exists, then potentially an enormous number of retired athletes would be at risk. ${ }^{6}$ Based on the published case studies, however, one estimate is that fewer than $4 \%$ of retired US professional football players may be at risk for this condition $^{7}$ rather than all exposed athletes, raising the issue that this may not be a part of impact exposure but rather due to other as yet unidentified factors.
A recent report by McKee et $a l^{8}$ has suggested that chronic traumatic encephalopathy (CTE) may represent a unique tauopathy with characteristic pathological stages; however, the published methodology does not allow a causal relationship to be determined between concussion or subconcussive impacts being a risk factor for CTE.

The purpose of this paper was to review the current state of evidence for CTE in retired athletes and to consider the potential differential diagnoses that require consideration when retired athletes present with cognitive and psychiatric problems.

\section{METHODS}

Articles were retrieved via online database searching, hand-searching reference lists and cited reference searches. The online databases of MEDLINE, CINAHL, EMBASE, Mosby's Index, PsycEXTRA, PsycINFO and Scopus were searched. Key words, $\mathrm{MeSH}$ terms and combinations of these were used to systematically search the databases. Key words included CTE, dementia pugilistica, punch drunk syndrome, traumatic encephalopathy, CTE, repetitive head injury, sports concussion, multiple concussions, chronic concussions, subconcussive blow and sports-related traumatic brain injury (TBI).

\section{CLINICAL SYNDROMES OF LONG-TERM PROBLEMS FOLLOWING CONCUSSION}

The clinical characterisation of the presentations of athletes with chronic postconcussive symptoms is poorly defined and may reflect intrinsic differences (eg, genetic) between individuals rather than the oversimplified understanding that these syndromes are due to concussive or subconcussive trauma alone. In some cases, the persistent 'postconcussive' symptoms may, in large part, be due to unrecognised depression or anxiety, ${ }^{9}$ which may be labelled as 'overtraining' or even athlete 'burnout'. ${ }^{10}$

If we assume concussion is the acute clinical syndrome with $90-95 \%$ of cases recovering in less than 10 days, then there is a small group where prolonged postconcussive symptoms exist, that is, where the symptoms persist more than 10 days after a single episode of acute concussion $(5-10 \%$ of cases) but full recovery eventually ensues usually within a matter of weeks. ${ }^{11}$ The clinical and neuropsychological features are those of a resolving acute injury and perhaps this entity should be considered as part of the acute syndrome.

In addition to the acute syndrome, a number of distinct but as yet poorly defined clinical subsets exist that may shed light on the process of recovery 
from concussive injury. These subsets are based on clinical experience and are not supported by hard scientific evidence at this time. Nevertheless, it may be useful to consider the full spectrum of presentations of athletes with persistent or permanent symptoms and the terminology of these states.

These include:

- Prolonged postconcussive symptoms as discussed above.

- Persistent postconcussion symptoms after one or more concussions where recovery is slow and may take months to years. ${ }^{12}$ The DSM-IV criteria for Post Concussion Syndrome is invoked only if the symptoms last beyond 3 months. Approximately $80 \%$ of such patients recover fully with time. ${ }^{13}$ Cognitive testing reveals mainly attentional deficits, and structural neuroimaging is normal.

- Permanent PCS - as per above, but these individuals do not recover fully, and this has been estimated at between $10 \%$ and $20 \%$ of all cases of persistent concussion symptoms. Functional MR and/or electrophysiological change may be present, but normal structural neuroimaging is typical. $^{14}$

- Proposed CTE where chronic cognitive and/or neurobehavioural dysfunction exists and a pathological diagnosis is subsequently confirmed. ${ }^{3}{ }^{6}$ It is worth noting that there are cases where the clinical phenotype is the same as pathologically confirmed CTE cases; however, no neuropathological change is demonstrated at autopsy. ${ }^{8}$

As well as these broad categories, there are other athletes who de novo manifest mental health issues (depression, anxiety, suicide) or neurobehavioural problems in the absence of persistent or prolonged concussive symptoms dating from the time of an injury. Whether these represent a variant of the subsets above or simply reflect the high incidence of such disorders in society remains to be elucidated. The risk factors for such complications following concussion remain unclear, although they may be related to repeated concussions, at least in retrospective surveys. $^{2} 15$ Prospective studies of head-injured individuals, with neuropathological and clinical verification, are needed to improve understanding of head trauma as a risk factor for sequelae. $^{16}$

As well as clinical symptoms, there is some limited objective evidence of persistent neurophysiological, cognitive ${ }^{16}$ and radiological ${ }^{17}$ deficits up to 30 years following concussion.

\section{PHYSIOLOGY OF CONCUSSION}

Understanding the pathophysiology of concussion would be expected to lead to an improvement in the assessment of deficit and recovery following injury, and to facilitate the accurate classification of severity. For example, little is known about the anatomical localisation of common clinical features such as headache, loss of consciousness (LOC), difficulty with concentration, sleep disturbance and fatigue. Moreover, it is currently unknown whether mild TBI reflects a single clinical entity with a linear spectrum of injury or even distinct injury subtypes. It is hoped that this understanding may give some insight into whether in vivo diagnosis of CTE is possible.

In animal models following acute injury, the release of neurotransmitters and ionic fluxes occurs (known as the 'neurometabolic cascade'), ${ }^{18}$ which in turn leads to changes in cell membrane function. Animal studies suggest that, during the glucose metabolic depression phase (1-10 days postinjury), the brain is more vulnerable to repeat injury. ${ }^{18}$ Changes in the intracellular fluid status or the presence of axonal swelling may be detected using imaging techniques such as advanced diffusion weighted imaging (DWI), which also allow mapping of white matter fibre tracts in the central nervous system. ${ }^{19}$ Preliminary studies have demonstrated DWI changes in the acute setting following mild TBI in a small cohort of adolescent patients with normal CT scans as well as in a cohort of asymptomatic professional boxers. ${ }^{20}$ Functional neuroimaging techniques, for example, functional MRI (fMRI) have demonstrated changes in brain function following sport-related TBI. ${ }^{21}$ The main limitation of this technique is that it only reveals regions of the brain that are active in the specific cognitive task being studied. Another imaging modality, Functional Connectivity, is able to detect realtime resting state networks and may provide an alternative to fMRI for identifying brain subregions and networks that are affected in mild TBI. ${ }^{22}$ Other techniques such as MR spectroscopy (MRS) allow the detection of metabolic disturbances following mild TBI through the measurement of intracellular metabolites. Preliminary studies in a small cohort of collegiate athletes suggest a role of mitochondrial dysfunction in the postinjury metabolic depression. ${ }^{23}$ Other studies using MRS have also demonstrated that the $\mathrm{N}$-acetylaspartate/creatine ratio (which reflects neuronal cell damage) is related to injury severity and outcome even when white matter appears normal on MRI. $^{24}$

\section{DEMENTIA RATES IN THE GENERAL POPULATION}

Given that cognitive impairment is one of the key features of the proposed CTE, it is important to understand the risk of this in similar age groups in the general population. The reported incidence and prevalence rates of dementia vary according to the population studied, and prevalence approximately doubles every 5 years from the age of 65 years. The incidence of dementia (all causes) in the 30-year-old to 64-year-old group is 54/ 100000 , and for the 45-year-old to 64-year-old group, it is 98.1: $100000 .^{25}$

Whether mild, or repetitive mild TBI (mTBI) increases an individual's risk for developing Alzheimer's disease (AD) has been a long-standing topic of contention. While mixed results have been reported regarding the association between moderate and severe TBI and AD, the association between $\mathrm{mTBI}$ and $\mathrm{AD}$ appears to be less strong. For example, in a systematic review, Bazarian et $a l^{26}$ concluded that there was limited support for the notion that mTBI (with LOC) results in an increased risk for later life AD. Further, the authors also concluded that there was insufficient evidence to determine whether an association between $\mathrm{mTBI}$ (without LOC) and AD exists.

\section{DEPRESSION IN THE GENERAL POPULATION}

Depression and cognitive impairment are both common conditions in older age, and they frequently occur together. A US-based epidemiology study examining major depression reported that the incidence of this disorder in men aged 25-34, $35-44$ and $45-54$ years was $12.3 \%, 11.0 \%$ and $8.6 \%$, respectively. ${ }^{27}$ This is much higher among individuals with dementia, where it has been reported that $25-50 \%$ of all patients with dementia will develop depression at some point over the course of their illness. 2829

\section{DEPRESSION, COGNITIVE IMPAIRMENT AND NEURODEGENERATIVE DISEASES IN FORMER ATHLETES}

In a study of retired National Football League (NFL) players, responses to questions regarding clinical depression revealed that $269(11.1 \%$; 95\% CI 9.9\% to $12.3 \%)$ of 2434 respondents reported a previous diagnosis of clinical depression. In comparison to retired NFL players with no history of concussion, retired players with a history of one or two previous 
concussions were 1.5 times more likely to be diagnosed with depression, while those with a history of three or more previous concussions were found to be three times more likely to be diagnosed with depression. ${ }^{15}$

The results of a screening survey of 513 retired NFL players (average age $=61$ years) reported that $35 \%$ produced scores suggesting a possibly mild cognitive impairment. ${ }^{2}$ In another recent study of former NFL players, the death rate from neurodegenerative diseases was three times greater than that of the general population, although the number of cases in this study was small. More specifically, the rates of diagnosis of $\mathrm{AD}$ and amyotrophic lateral sclerosis (ALS) were found to be four times higher in former NFL players than the general population. ${ }^{30}$ Of the 334 former athletes in this cohort, seven (2.1\%) had ALS listed on their death certificates. This issue of ALS is in keeping with the TDP43 tauopathy seen in CTE cases. ${ }^{31}$

\section{BRAIN PATHOLOGY IN NORMAL AGEING}

The Honolulu-Asia Aging Study provides a unique longitudinal model of ageing and disease and offers considerable insight into what may be considered normal ageing from a pathological standpoint. $^{32}$ From 1991 to 1993,3 yearly follow-up clinical and formal neuropsychological examinations have been conducted in 3508 men who were free of dementia and a total of 593 standardised brain autopsies have been conducted. ${ }^{32}$

Postmortem brain examination demonstrated diverse pathology, even in individuals clinically diagnosed as 'pure AD,' with fewer than $50 \%$ demonstrating the typical pathological features of AD. Furthermore, neuropathological abnormalities were also observed in approximately $40 \%$ of neuropsychologically normal patients. ${ }^{32-34}$ Similarly, disparity between clinical presentation (ie, living diagnosis) and postmortem neuropathology has also been demonstrated in other ageing study samples. ${ }^{35} 36$ It is now clear from these ageing studies that postmortem findings may not represent pathology and may not equate to clinical symptomology or a syndrome ${ }^{37}$ and may be seen in cognitively normal older adults. Whether the 'gold standard' of neuropathology in neurological disease is true has been recently questioned. ${ }^{38}$

\section{THE 'CLASSIC' SYNDROME OF CTE IN PROFESSIONAL BOXERS}

In 1928, Dr Harrison Martland first described the 'punch drunk' or CTE state in retired boxers. ${ }^{39}$ The incidence of classic CTE has proven difficult to establish, due, in the main, to a lack of prospective studies. Roberts, ${ }^{40}$ who randomly sampled 250 retired boxers from a cohort of 16781 UK boxers registered between 1929 and 1955, reported that in 37 boxers (17\%) clinically demonstrable lesions of the nervous system were present. Notably, the oldest boxers in this cohort fought in the late 1800 s, in an era where bare-knuckle championships were still conducted, frequent fights occurred even when boxers were concussed and there was little medical supervision or weight matching of boxers; however, only 11 of the 37 cases were elaborated on in this study. A close analysis of the clinical details raises suspicion regarding the certainty of the diagnosis of CTE in most cases. ${ }^{41}$

In the published cases, cognitive deterioration was typically detected 10-20 years subsequent to cessation of exposure to repetitive head trauma (ie, postretirement). ${ }^{6}$ Interestingly, in all cases where details were provided, the physical signs but not the cognitive deficits progressed postretirement. There are two distinct clinical syndromes that have been demonstrated in this data set; the first (which occurs in approximately $70 \%$ of cases) includes dysarthria, pyramidal problems and cognitive deficits.
As the disease manifests clinically, these cognitive abnormalities include difficulties in memory, information processing speed, insight and orientation. The second clinical syndrome (in approximately $30 \%$ of cases) includes dysarthria and pyramidal problems, but with intact cognitive abilities. ${ }^{40-44}$ Movement disorders were reported to be present in approximately two in every five reported cases. ${ }^{3}$

One of the difficulties of ascribing the clinical syndrome solely to boxing is the presence of comorbidities plus risk factors for other conditions that may also result in cognitive deterioration. A case report of a champion boxer with cognitive decline highlights these issues of multifactorial causation of cognitive problems. ${ }^{45}$

The neuropathological features of classic CTE have been described in detail ${ }^{46}$ and typically result in a cavum septum pellucidum with septal fenestration; cerebellar scarring involving Purkinje cell loss and thinning of the granular layer; degeneration of the substantia nigra and locus caeruleus; and diffuse neurofibrillary tangles (NFT) involving the medial temporal region, uncus, amygdala, hippocampus, parahippocampal gyrus and fusiform gyrus along with the more lateral temporal, insular and frontal cortices. The extent of neuropathology appears to be positively correlated with the level of exposure. ${ }^{46}$ Roberts et $a l^{44}$ examined $14 / 15$ brains originally described by Corsellis et $a l^{46}$ as well as six additional boxers' brains using immunocytochemistry, and 19/21 cases also demonstrated widespread diffuse amyloid deposits.

Recently, a propagation model of neurodegeneration has been proposed, suggesting that tau positive NFT phosphorylation may progressively spread from one neuron to adjacent neurons in the absence of ongoing triggering factors. ${ }^{47-50}$ The implication of this finding is that the neuropathological findings may differ or progress in different stages of the condition. This finding may help resolve the differences between reports of specific sites of tau positive NFT deposition in CTE and other studies that suggest widespread changes.

\section{THE 'MODERN' SYNDROME OF CTE IN FOOTBALLERS}

Recent publications ${ }^{3}{ }^{51}$ on CTE in retired athletes have introduced a number of conceptual changes in the clinical features $^{52} 53$ and outcomes, and also the neuropathology, as compared with the classic entity described by Roberts ${ }^{40}$ and Corsellis et $a l^{46}$ in their boxing subjects.

\section{PUBLISHED MODERN CTE CASES}

A number of cases of CTE in retired athletes have been published. The index case was reported by Omalu and colleagues in 2005, with additional cases reported subsequently. ${ }^{4} 51 \quad 54-56$ Recently, the Boston University group published their experience of CTE, with 80 athlete brain donors (22 of whom were also military veterans) with a history of repetitive brain injury, and they found that $80 \%$ of these cases demonstrated the characteristic pathology for CTE. ${ }^{8}$

\section{Signs and symptoms}

The modern CTE description suggests that symptoms such as gait disorders, speech slowing and extrapyramidal signs may be present; however, neuropsychiatric and behavioural symptoms tend to predominate early. ${ }^{57}$ The most common symptoms reported are mood disorder (mainly depression), paranoia, agitation, social withdrawal, poor judgement and aggression. Cognitive impairment tends to emerge as the major neuropsychiatric feature in the latter stages ${ }^{58}$ and typically includes impairment across the domains of orientation, memory, 
language, attention, information processing speed and executive functioning. ${ }^{60}$ These cognitive symptoms have been proposed to progress in a somewhat predictable manner, ${ }^{3}$ whereas the classic CTE entity reported little progression of cognitive deficits. ${ }^{46}$

\section{Neuropathology}

The reported neuropathological characteristics of both entities (classic and modern CTE) appear to be more closely related and share a number of common features such as fenestrated septum pellucidum, cerebral atrophy, tau+NFT inclusion (although found in greater amounts in modern CTE), $\beta$-amyloid deposition (found in less amounts in modern CTE), reduced pigmentation of the substantia nigra and locus caeruleus, and enlarged ventricles. The qualitative description of modern CTE characterises the neuropathological change as also including frontotemporal lobe atrophy with extensive tau pathology distributed throughout the neocortex, medial temporal lobe, diencephalon, brainstem and spinal cord. In the 51 cases reviewed by Gavett et $a l^{761}$ diffuse amyloid plaques were found in 24 (47\%), neuritic amyloid plaques in $13(27 \%)$ and amyloid angiopathy in $3(6 \%)$. In the Boston study, ${ }^{8}$ amyloid deposition was noted in $44 \%$ of the CTE cases.

Although a number of similarities in neuropathological findings have been reported across the modern CTE cases, there are also some important differences. McKee $e t a l^{3}$ have reported a marked accumulation of tau-immunoreactive astrocytes, but this has not been observed in any of the cases examined by Omalu et al. ${ }^{62}$ Omalu et al ${ }^{63}$ have reported the skipphenomenon, with a propensity to lobar cortical distribution in the absence of prominent periventricular topographic distribution, which has not been described in the McKee et $a l^{3}$ cases.

A defining neuropathological feature of the modern CTE entity is abundant filamentous tau lesions, of which the patterns of expression are considered unique, occurring in the absence (or at least relative scarcity) of $\beta$-amyloid deposits. ${ }^{3} 8$ Prominent filamentous tau inclusions and brain degeneration in the absence of $\beta$-amyloid deposits are also considered the sine qua non of a number of neurodegenerative tauopathies. It is worth noting that a number of the degenerative tauopathies, such as behavioural variant fronto-temporal dementia, share many of the clinical and pathological features of 'modern' CTE. ${ }^{64-69}$

\section{DISCUSSION}

The recent autopsy cases differ from the classic CTE description across a number of characteristics including age of onset, progression, assumed predominate (clinical) features and diagnostic criteria. It has also been reported that these recent cases of modern CTE, speculated to be a consequence of concussive and subconcussive blows, are characterised by a distinct neuropathological profile ${ }^{3} 6163$ and manifest primarily as a tauopathy. ${ }^{8}$ Although many of the reported macroscopic neuropathological features are common among the original and newer descriptions of CTE, the different distribution of tau-immunoreactive astrocytes distinguishes the newer description with preferential involvement of the superficial cortical layers occurring on a background of relative scarcity of $\beta$-amyloid plaques.

At present, there are no published epidemiological, crosssectional or prospective studies relating to modern CTE. Owing to the nature of the published studies, being case reports or pathological case series, it is not possible to determine the causality or risk factors with any certainty. As such, the speculation that repeated concussion or subconcussive impacts cause CTE remains unproven. ${ }^{70}$
The extent to which age-related changes, psychiatric or mental health illness, alcohol/drug use or coexisting dementing illnesses contribute to this process is largely unaccounted for in the published literature. In addition, consideration for the potential genetic risk in those athletes with a family history of neurodegenerative disease and the extent to which this contributes to the clinical and pathological profiles also require further investigation. ${ }^{41}$

\section{SUMMARY}

At present, the interpretation of causation in the modern CTE case studies should proceed cautiously. The causal assumptions require further prospective or longitudinal studies on the topic. Ultimately, scientific research might establish that participation in contact sports leads to a distinct neuropathological syndrome, and this neuropathology causes psychiatric, cognitive and physical problems, but this cause and effect relationship remains to be shown scientifically.

\section{Author affiliations}

${ }^{1}$ The Florey Institute of Neuroscience and Mental Health, Heidelberg, Australia ${ }^{2}$ Faculty of Kinesiology, Sport Injury Prevention Research Centre, Calgary, Alberta, Canada

${ }^{3}$ Faculty of Medicine, Hotchkiss Brain Institute, University of Calgary, Calgary, Alberta, Canada

${ }^{4}$ Department of Neurology, University of Michigan, Ann Arbor, Michigan, USA

${ }^{5}$ Burke Rehabilitation Hospital, White Plains, New York, USA

${ }^{6}$ Neuropsychiatry Service, HNE Mental Health Service, Newcastle, New South Wales, Australia

Competing interests See the supplementary online data for competing interests (http://dx.doi.org/10.1136/bjsports-2013-092248).

Provenance and peer review Commissioned; internally peer reviewed.

\section{REFERENCES}

1 Gregory $S$. The problem with football. Our favorite sport is too dangerous. How to make the game safer. Time 2010;175:36-43.

2 Guskiewicz KM, Marshall SW, Bailes J, et al. Association between recurrent concussion and late-life cognitive impairment in retired professional football players. Neurosurgery 2005;57:719-26.

3 McKee AC, Cantu RC, Nowinski CJ, et al. Chronic traumatic encephalopathy in athletes: progressive tauopathy after repetitive head injury. I Neuropathol Exp Neurol 2009;68:709-35.

4 Omalu BI, Hamilton RL, Kamboh MI, et al. Chronic traumatic encephalopathy (CTE) in a National Football League Player: case report and emerging medicolegal practice questions. J Forensic Nurs 2010;6:40-6.

5 Cantu RC. Chronic traumatic encephalopathy in the National Football League. Neurosurgery 2007;61:223-5.

6 McCrory P. Sports concussion and the risk of chronic neurological impairment. Clin J Sport Med 2011;21:6-12.

7 Gavett BE, Cantu RC, Shenton M, et al. Clinical appraisal of chronic traumatic encephalopathy: current perspectives and future directions. Curr Opin Neurol 2011;24:525-31.

8 McKee AC, Stein TD, Nowinski CJ, et al. The spectrum of disease in chronic traumatic encephalopathy. Brain 2012;136:43-64.

9 Chen JK, Johnston KM, Collie A, et al. A validation of the post concussion symptom scale in the assessment of complex concussion using cognitive testing and functional MRI. J Neurol, Neurosurg Psychiatry 2007;78:1231-8.

10 Schwenk TL, Gorenflo DW, Dopp RR, et al. Depression and pain in retired professional football players. Med Sci Sports Exercise 2007;39:599-605.

11 Makdissi M, Darby D, Maruff $P$, et al. Natural history of concussion in sport: markers of severity and implications for management. Am J Sports Med 2010;38:464-71

12 King N. Mild head injury: neuropathology, sequelae, measurement and recovery. $\mathrm{Br}$ J Clin Psychol/Br Psychol Soc 1997;36(Pt 2):161-84.

13 Sullivan KA, Edmed SL, Cunningham LC. A comparison of new and existing mild traumatic brain injury vignettes: recommendations for research into post-concussion syndrome. Brain Inj 2013;27:19-30.

14 King NS, Kirwilliam S. Permanent post-concussion symptoms after mild head injury. Brain Inj 2011;25:462-70.

15 Guskiewicz KM, Marshall SW, Bailes J, et al. Recurrent concussion and risk of depression in retired professional football players. Med Sci Sports Exercise 2007;39:903-9. 
16 De Beaumont $\mathrm{L}$, Theoret $\mathrm{H}$, Mongeon $\mathrm{D}$, et al. Brain function decline in healthy retired athletes who sustained their last sports concussion in early adulthood. Brain 2009:132(Pt 3):695-708

17 Kraus MF, Susmaras T, Caughlin BP, et al. White matter integrity and cognition in chronic traumatic brain injury: a diffusion tensor imaging study. Brain 2007; 130(Pt 10):2508-19.

18 Giza CC, Hovda DA. The neurometabolic cascade of concussion. J Ath/ Train 2001;36:228-35

19 Metting Z, Rodiger LA, De Keyser J, et al. Structural and functional neuroimaging in mild-to-moderate head injury. Lancet Neurol 2007;6:699-710.

20 Zhang L, Heier LA, Zimmerman RD, et al. Diffusion anisotropy changes in the brains of professional boxers. Am J Neuroradiol 2006:27:2000-4.

21 Chen JK, Johnston KM, Collie A, et al. Behavioural and functional imaging outcomes in symptomatic concussed athletes measured with cogsport and functional MRI. BJ Sport Med 2004;38:659.

22 Waites $A B$, Briellmann RS, Saling MM, et al. Functional connectivity networks are disrupted in left temporal lobe epilepsy. Ann Neurol 2006;59:335-43.

23 Vagnozzi R, Signoretti S, Tavazzi B, et al. Temporal window of metabolic brain vulnerability to concussion: a pilot $1 \mathrm{H}$-magnetic resonance spectroscopic study in concussed athletes_-part III. Neurosurgery 2008;62:1286-95.

24 Garnett MR, Blamire AM, Corkill RG, et al. Early proton magnetic resonance spectroscopy in normal-appearing brain correlates with outcome in patients following traumatic brain injury. Brain 2000;123(Pt 10):2046-54.

25 Harvey RJ, Skelton-Robinson M, Rossor MN. The prevalence and causes of dementia in people under the age of 65 years. J Neurol Neurosurg Psychiatry 2003;74:1206-9.

26 Bazarian JJ, Cernak I, Noble-Haeusslein L, et al. Long-term neurologic outcomes after traumatic brain injury. J Head Trauma Rehabil 2009;24:439-51.

27 Gonzalez HM, Tarraf W, Whitfield KE, et al. The epidemiology of major depression and ethnicity in the United States. J Psychiatr Res 2010;44:1043-51.

28 Olin JT, Katz IR, Meyers BS, et al. Provisional diagnostic criteria for depression of Alzheimer disease: rationale and background. Am J Geriatr Psychiatry 2002;10:129-41.

29 Steinberg EM, Rubinow DR, Bartko JJ, et al. A cross-sectional evaluation of perimenopausal depression. J Clin Psychiatry 2008;69:973-80.

30 Lehmann EJ, Hein MJ, Baron SL, et al. Neurodegenerative causes of death among retired National Football League players. Neurology 2012;79:1-5.

31 McKee AC, Gavett BE, Stern RA, et al. TDP-43 proteinopathy and motor neuron disease in chronic traumatic encephalopathy. J Neuropathol Exp Neurol 2010;69:918-29.

32 White L. Brain lesions at autopsy in older Japanese-American men as related to cognitive impairment and dementia in the final years of life: a summary report from the Honolulu-Asia aging study. J Alzheimers Dis 2009;18(Pt 3):713-25.

33 Robinson JL, Geser F, Corrada MM, et al. Neocortical and hippocampal amyloid-beta and tau measures associate with dementia in the oldest-old. Brain 2011;134:3708-15

34 Gelber RP, Launer LJ, White LR. The Honolulu-Asia Aging Study: epidemiologic and neuropathologic research on cognitive impairment. Curr Alzheimer Res 2012;9:664-72.

35 Snowden JS, Gibbons ZC, Blackshaw A, et al. Social cognition in frontotemporal dementia and Huntington's disease. Neuropsychologia 2003;41:688-701.

36 Sonnen JA, Larson EB, Crane PK, et al. Pathological correlates of dementia in longitudinal, population-based sample of aging. Ann Neurol 2007;62:406-13.

37 Da Cruz S, Cleveland DW. Understanding the role of TDP-43 and FUS/TLS in ALS and beyond. Curr Opin Neurobiol 2011;21:904-19.

38 Scheltens $\mathrm{P}$, Rockwood K. How golden is the gold standard of neuropathology in dementia? Alzheimers Demen 2011;7:486-9.

39 Martland HS. Punch drunk. J Am Med Assoc 1928:19:1103-7.

40 Roberts $\mathrm{AH}$. Brain damage in boxers: a study of the prevalence of traumatic encephalopathy among ex-professional boxers. London: Pitman, 1969.

41 McCrory P. Boxing and the risk of chronic brain injury. BMJ 2007:335:781-2.

42 Johnson J. Organic psychosyndromes due to boxing. Br J Psychiat 1969;115:45-53.

43 Johnson J. The EEG in the traumatic encephalopathy of boxers. Psychiatr Clin $1969 ; 2: 204-11$.
44 Roberts GW, Allsop D, Bruton C. The occult aftermath of boxing. J Neurol Neurosurg Psychiatry 1990;53:373-8.

45 Nowak LA, Smith GG, Reyes PF. Dementia in a retired world boxing champion: case report and literature review. Clin Neuropathol 2009;28:275-80.

46 Corsellis JA, Bruton CJ, Freeman-Browne D. The aftermath of boxing. Psychol Med 1973:3:270-303.

47 De Calignon A, Polydoro M, Suarez-Calvet $\mathrm{M}$, et al. Propagation of tau pathology in a model of early Alzheimer's disease. Neuron 2012;73:685-97.

48 Liu L, Drouet V, Wu JW, et al. Trans-synaptic spread of tau pathology in vivo. PLoS ONE 2012:7:e31302.

49 Frost B, Jacks RL, Diamond MI. Propagation of tau misfolding from the outside to the inside of a cell. J Biol Chem 2009;284:12845-52.

50 Clavaguera F, Goedert M, Tolnay M. Induction and spreading of tau pathology in a mouse model of Alzheimer's disease. Med Sci 2010;26:121-4.

51 Omalu BI, Bailes J, Hammers JL, et al. Chronic traumatic encephalopathy, suicides and parasuicides in professional American athletes: the role of the forensic pathologist. Am J Forensic Med Pathol 2010;31:130-2.

52 Clausen $\mathrm{H}$, McCrory $\mathrm{P}$, Anderson V. The risk of chronic traumatic brain injury in professional boxing: change in exposure variables over the past century. $\mathrm{Br} J$ Sports Med 2005;39:661-4.

53 Ahmed OH, Sullivan SJ, Schneiders AG, et al. iSupport: do social networking sites have a role to play in concussion awareness? Disabil Rehabil 2010 32:1877-83.

54 Omalu BI, DeKosky ST, Hamilton RL, et al. Chronic traumatic encephalopathy in a national football league player: part II. Neurosurgery 2006;59:1086-92; discussion 92-3.

55 Omalu BI, DeKosky ST, Minster RL, et al. Chronic traumatic encephalopathy in a National Football League player. Neurosurgery 2005;57:128-34; discussion 34.

56 Omalu BI, Fitzsimmons RP, Hammers J, et al. Chronic traumatic encephalopathy in a professional American wrestler. J Forensic Nurs 2010:6:130-6.

57 Jordan B. Neurologic aspects of boxing. Arch Neurol 1987;44:453-9.

58 Guterman A, Smith RW. Neurological sequelae of boxing. Sports Med (Auckland, NZ) 1987;4:194-210.

59 Jordan BD. Chronic traumatic brain injury associated with boxing. Semin Neurol 2000;20:179-85

60 Costanza A, Weber K, Gandy S, et al. Review: contact sport-related chronic traumatic encephalopathy in the elderly: clinical expression and structural substrates. Neuropathol App/ Neurobiol 2011;37:570-84.

61 Gavett BE, Stern RA, McKee AC. Chronic traumatic encephalopathy: a potential late effect of sport-related concussive and subconcussive head trauma. Clin Sports Med 2011:30:179-88, xi.

62 Omalu B, Hammers JL, Bailes J, et al. Chronic traumatic encephalopathy in an Iraqi war veteran with posttraumatic stress disorder who committed suicide. Neurosurg Focus 2011;31:E3

63 Omalu B, Bailes J, Hamilton RL, et al. Emerging histomorphologic phenotypes of chronic traumatic encephalopathy in American athletes. Neurosurgery 2011:69:173-83; discussion 83.

64 Rascovsky K, Salmon DP, Ho GJ, et al. Cognitive profiles differ in autopsy-confirmed frontotemporal dementia and AD. Neurology 2002;58:1801-8.

65 Rascovsky K, Hodges JR, Knopman D, et al. Sensitivity of revised diagnostic criteria for the behavioural variant of frontotemporal dementia. Brain 2011; 134(Pt 9):2456-77.

66 Rascovsky K, Hodges JR, Kipps CM, et al. Diagnostic criteria for the behavioral variant of frontotemporal dementia (bvFTD): current limitations and future directions. Alzheimer Dis Assoc Disord 2007;21:S14-8.

67 Hodges JR. Frontotemporal dementia (Pick's disease): clinical features and assessment. Neurology 2001;56(11 Suppl 4):S6-10.

68 Hodges JR, Davies RR, Xuereb JH, et al. Clinicopathological correlates in frontotemporal dementia. Ann Neurol 2004;56:399-406.

69 Hodges JR, Miller B. The classification, genetics and neuropathology of frontotemporal dementia. Introduction to the special topic papers: part I. Neurocase 2001;7:31-5.

70 Gaetz W, Macdonald M, Cheyne D, et al. Neuromagnetic imaging of movement-related cortical oscillations in children and adults: age predicts post-movement beta rebound. Neuroimage 2010;51:792-807. 